\title{
Paramagnetic spherical nanoparticles by self-assembly of persistent trityl radicals.
}

\author{
I. Marin-Montesinos, ${ }^{a}$ J. C. Paniagua ${ }^{b, c}$ Alejandro Peman, ${ }^{a}$ M. Vilaseca ${ }^{d}$, F. Luis, ${ }^{e}$ S. Van Doorslaer, ${ }^{f}$ and M. Pons ${ }^{a}$
}

Spherical nanoparticles and fibres observable by cryo-electron microscopy are spontaneously formed by the Finland trityl radical at concentrations above $15 \mathrm{mM}$. These species represent a new class of paramagnetic, metal-free, nanoscale supramolecular materials. Selfassociation was observed under a variety of experimental conditions, including aqueous solution at room temperature, low temperature frozen glasses and the gas phase. Oligomers formed by at least 5 Finland radicals were detected by ion-mobility mass spectrometry. Magnetic susceptibility data as well as low temperature EPR spectra show coupling between electronic spins in the self-assembled species. Quantum chemical calculations show stacking along the C3 symmetry axis. Nanoparticle formation requires additional lateral packing that can be provided by bydrogen bonding involving the triangular array of carboxylic acid groups leading to the assembly of geodesic spheres.

\section{Introduction}

High spin organic molecules are attracting a great deal of interest as building blocks for organic magnetic materials, ${ }^{1}$ as paramagnetic relaxation agents, ${ }^{2}$ and for spintronics. ${ }^{3}$ Covalent polyradical species are being actively produced for these applications. ${ }^{4}$ Functional molecular materials based on organic radicals require processing techniques to organize stable radicals into films or size-controlled nanostructures. ${ }^{1}$ Organization of radicals on low dimensionality systems is specially attractive from the applications point of view. For example single-layer magnetic films may represent a limit for the miniaturization of data storage devices, ${ }^{5}$ and hold potential in molecular electronics, optoelectronics and sensors. ${ }^{6}$ Radical deposition on gold surfaces or graphene have been demonstrated. $^{7}$ Functionalization of single-wall carbon nanotubes has been suggested as a strategy to obtain materials amenable to composite formation. ${ }^{8}$ Self-association of persistent radicals, without an external scaffold, offers an alternative route to polyradicals.

Self-assembly of suitably decorated organic frameworks is a rich theme in supramolecular chemistry. The conformational restriction present in calixarenes, or resorcinarenes has been used to direct hydrogen bonding of pendant groups, e.g. ureas, leading to self-assembly into dimers or larger species. ${ }^{9}$ Trityl groups containing bulky substituents show considerable conformational restriction. A striking example is the slow interconversion between atropoisomers of perchlorinated triarylmethane derivatives. ${ }^{10}$

Tetrathiatriarylmethyl (TAM) radicals are an important class of persistent trityl radicals. ${ }^{11}$ The Finland radical (tris-(8carboxyl-2,2,6,6-tetramethyl-1,3,5,7-tetrathia-2,6-dihydro-sindacene-4-yl)methyl sodium salt) (Scheme 1) is the simplest member of the series. The chemical stability of TAM radicals is the result of electron delocalization and steric crowding caused by the presence of bulky sulfur groups. Water-soluble TAM radicals, containing charged carboxylate groups, have found numerous biological applications. Trityl radicals give sharp Electron Paramagnetic Resonance (EPR) signals and are sensitive in vivo reporters in EPR based oxymetry and probes for the presence of reactive oxygen species. ${ }^{12}$ Lately, they are being extensively used as additives to increase NMR sensitivity by dynamic nuclear polarization (DNP), ${ }^{13,14}$ especially in the context of Magnetic Resonance Imaging (MRI) for metabolic imaging potentially leading to new cancer diagnostic tools. ${ }^{15}$

In a previous study we explored the supramolecular properties of OX63, a related TAM radical, widely used in DNP, formerly considered to exist as non-interacting monomers in solution. We showed that OX63 formed dimers with stabilizing hydrogen bonds between the hydroxyethyl group. ${ }^{16}$ In the present work we explore the supramolecular properties of the simpler Finland radical, in which hydroxyethyl groups are replaced by methyl. We find that Finland radical selfassociation is maintained. However, it does not stop at the dimer level but, at concentrations above $15 \mathrm{mM}$, spherical nanoparticles and fibers are observed by cryo-electron microscopy (EM).

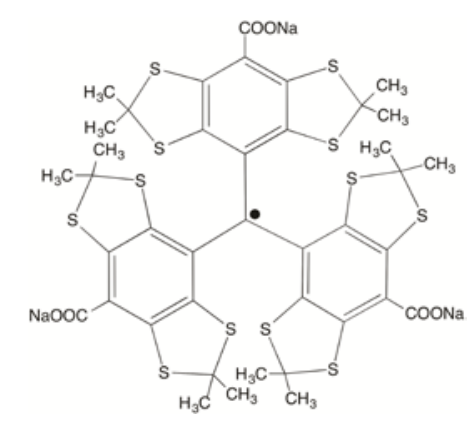

Scheme 1. The Finland radical. (tris-(8-carboxyl-2,2,6,6-tetramethyl-1,3,5,7tetrathia-2,6-dihydro-s-indacene-4-yl)methyl sodium salt)

\section{Results and discussion}

UV-vis spectra of Finland radicals were measured in phosphate buffer, $\mathrm{pH} 7.0$, over a concentration range from $5 \mu \mathrm{M}$ to $5 \mathrm{mM}$ using cells with path lengths from $1 \mathrm{~cm}$ to $0.01 \mathrm{~cm}$. Representative spectra are shown in the suporting information (SI). While no changes in the position of the absorption bands 
were observed, the apparent molar absorptivity ( $\left.\varepsilon_{\text {app}}\right)$ decreases by about $30 \%$ with increasing concentration. The decrease takes place for concentrations above $60 \mu \mathrm{M}$ in two steps, separated by a short plateau (Figure 1). These results show the self-association of Finland dimers above micromolar concentrations. Hypochromicity was correctly predicted by time dependent DFT calculations described below, and has been observed in the interaction of other chromophores. ${ }^{17}$

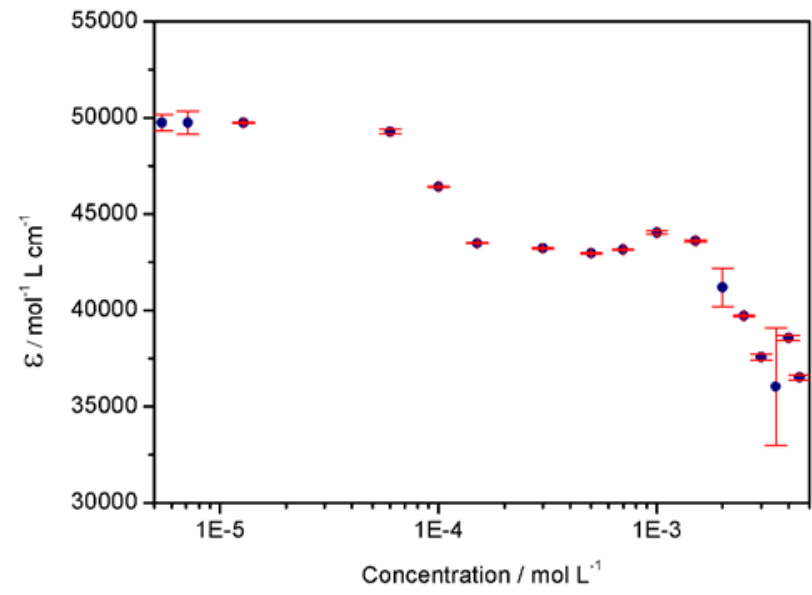

Figure 1. Concentration dependence of the apparent molar absorptivity at 271 $\mathrm{nm}$ of Finland radical solutions in phosphate buffer at pH 7.0 and $298 \mathrm{~K}$.

The macroscopic paramagnetic response of concentrated Finland solutions evidences also the formation of supramolecular agglomerates. Figure 2 shows magnetization isotherms (main panel) and the low-field susceptibility (inset) of a $20 \mathrm{mM}$ solution in 1:1 glycerol-water. While the former agree reasonably well with the response expected for free $S=1 / 2$ radicals, the latter evidences the presence of high-spin $(S>1 / 2)$ magnetic species. The theoretical susceptibility curves for various $\mathrm{S}$ values are shown along with the experimental susceptibilities in SI figure S2. The fact that magnetization and susceptibility curves point to different average spin values is characteristic of a sample with a distribution of molecular species with different spin values. ${ }^{18}$

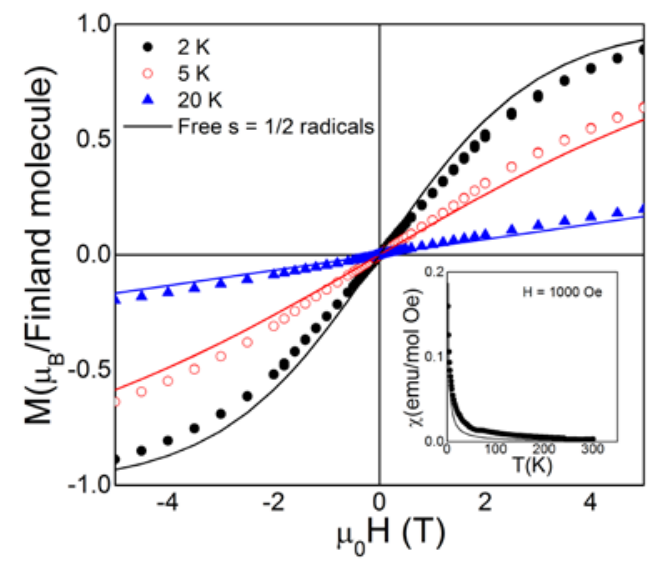

Figure 2. Magnetization (main panel) and low-field susceptibility (inset) measurements of $20 \mathrm{mM}$ Finland in 1:1 glycerol-water. The solid curves in both panels show the expected response of non-interacting radicals.

In addition, the magnetic response depends on the speed at which the sample is frozen from room temperature (SI, Figure S2), thus suggesting that different supramolecular aggregates might also have different stabilization energies.

Figure 3 shows CW and electron spin echo (ESE) detected Xband EPR spectra of frozen solutions of Finland radicals at varying concentrations. CW EPR spectra were measured at 1.5 $\mathrm{mM}, 15 \mathrm{mM}$ and $30 \mathrm{mM}$ concentrations in glycerol- $\mathrm{D}_{2} \mathrm{O}$ (1:1) at $20 \mathrm{~K}$ in the presence of tetramethylammonium (TMA) for direct comparison with published spectra in OX63. ESEdetected EPR spectra were measured at $7 \mathrm{~K}$ in glycerol- $\mathrm{H}_{2} \mathrm{O}$ without TMA. CW-EPR spectra of solutions without TMA are shown in SI figure S3.

The CW-EPR spectra of the Finland radical (Figure 3a) are dominated by a narrow central signal at $g=2.0030$ with side bands. At low concentration $(1.5 \mathrm{mM})$ only a pair of side bands, separated by about $5 \mathrm{G}$ is observed. Similar side-bands were observed in frozen solution ${ }^{19}$ and were assigned to ${ }^{13} \mathrm{C}$ hyperfine couplings and spin-flip satellite lines. ${ }^{19,20}$ At $15 \mathrm{mM}$ and $30 \mathrm{mM}$ additional side bands appear: a pair of intense broad peaks with a splitting $\sim 60 \mathrm{MHz}(2.2 \mathrm{mT}$ ) (indicated by dashed arrows in Figure 3 ) and a weaker pair split by $\sim 122$ $\mathrm{MHz}$ (4.4 mT) (solid arrows in Figure 3). The relative intensity of these side bands with respect to the central band clearly increases with radical concentration, thus suggesting that they originate from radical clusters (dimers or higher order oligomers). The splitting pattern suggests the presence of dipolarly interacting radicals. In line with this observation, halffield EPR signals originating from $\left|\Delta \mathrm{M}_{\mathrm{S}}\right|=2$ transitions were observed at $15 \mathrm{mM}$ and $30 \mathrm{mM}$ concentrations, implying $\mathrm{S} \geq 1$ systems and thus confirming the presence of radical clusters (Inset of Fig. 3). The intensity of the half-field signals decreased with concentration. The fact that the side bands change intenstity, but not their position, upon dilution allows to rule out the hypothesis that coupling merely occurs between non-interacting radicals that are located close enough in a random distribution of radicals at high concentration. In addition, such random approaches would be expected to give a distribution of couplings but not sharp side bands.

In order to corroborate the above assignment, X-band pulsed EPR experiments were performed. These confirmed the presence of $S>1 / 2$ systems at higher concentrations of the Finland radical. Firstly, the optimal tuning of the microwave pulses at $7 \mathrm{~K}$ required a considerably lower $\mathrm{B}_{1}$ (higher pulse attenuation) in the case of the $30 \mathrm{mM}$ Finland frozen solution than for the $1.5 \mathrm{mM}$ Finland frozen solution. While the pulse tuning in the latter case (3dB) was similar to that of a standard coal sample ( $2 \mathrm{~dB})$, the high pulse attenuation $(16 \mathrm{~dB})$ of the 30 $\mathrm{mM}$ Finland frozen solution indicates the presence of high-spin systems.

Second, the ESE-detected EPR spectra confirm the CW-EPR observation that additional side bands are present in the higher 
concentrated sample (Figure $3 b$ ). The width and form of the extra side-band pattern cannot be explained in terms of a hyperfine pattern, but is due to Pake pattern-type contributions of dipolarly interacting radicals with inter-spin distances 10.1 and 11.0 angstrom (SI Figure S4). The side bands are not due to random clustering because of the high radical concentration, since this would lead to a broad background signal as opposed to the well-defined Pake patterns observed here. Moreover, changing of the radical concentration would then lead to changed inter-spin distances. Here, a lowering of the concentration from 30 to $15 \mathrm{mM}$ leads to a reduction of the side band intensity, but not in a shift of the peak positions (Figure 3a).

Finally, PEANUT experiments ${ }^{21}$ were performed at the central magnetic field position and on two magnetic fields in the side bands (SI, Figure S5). The shift of the nutation frequency confirms that the side bands stem from high-spin (mainly $S=1$ ) systems. The presence of higher-spin systems, as suggested by magnetization experiments, that are not directly observed by EPR due to fast relaxation cannot be ruled out.

The geometry of Finland dimers was investigated using DFT calculations performed with the ORCA package. ${ }^{22}$ The BP functional $^{23}$ was used with the def2-SVP basis set. ${ }^{24}$ Solvent effects were introduced using the conductor like screening model (COSMO). ${ }^{25}$ A previous investigation of the Finland monomer using the B3LYP functional with a 6-31G basis set led to a $\mathrm{D}_{3}$ symmetrical propeller-shaped geometry. ${ }^{19}$ Our calculations show that a $8.6 \mathrm{kcal} / \mathrm{mol}$ more stable $\mathrm{C}_{3}$-symmetry dome structure exists in which the two sides of the propeller are non-equivalent as a result of differences in puckering of the five membered dithiole rings. The two sides are referred to as “open” and "closed”. A CC distance of $4.6 \AA$ in the closed side and $8.2 \AA$ in the open side separate the nearest methyl groups (Figure 4).

The open side of the dome conformation has 3 methyl groups with their CC bonds nearly parallel to the symmetry axis, and oriented in such a way that they can interdigitate with the equivalent groups of a second Finland molecule like the teeth of a zipper. This dome structure can adopt two enantiomeric propeller conformations, $\mathrm{P}$ (plus) and $\mathrm{M}$ (minus), and it can approach a second molecule by its open ("in”) or closed (“out”) side. Thus, several dimer geometries are possible. Table 1 collects their stabilization energies relative to the separated monomers, the distance between the two central $\mathrm{sp}^{2}$ carbons and a dihedral angle measuring the relative staggering of the two propellers in the dimer. The dimer PoutPin is not included because no stable geometry with a 3-fold symmetry axis was found. The structures of the dimers are shown in SI figure S6.

The lowest energy dimer has $\mathrm{S}_{6}$ symmetry and is non-chiral. Interdigitation of the methyl groups contributes to the van der
Waals stabilization of the dimers. The short (2.6 $\AA)$ H...S distances observed in the PinMin dimer (Figure 4) suggest a contribution from non-classical intermolecular hydrogen bonds between methyl groups and sulfur atoms. ${ }^{26}$
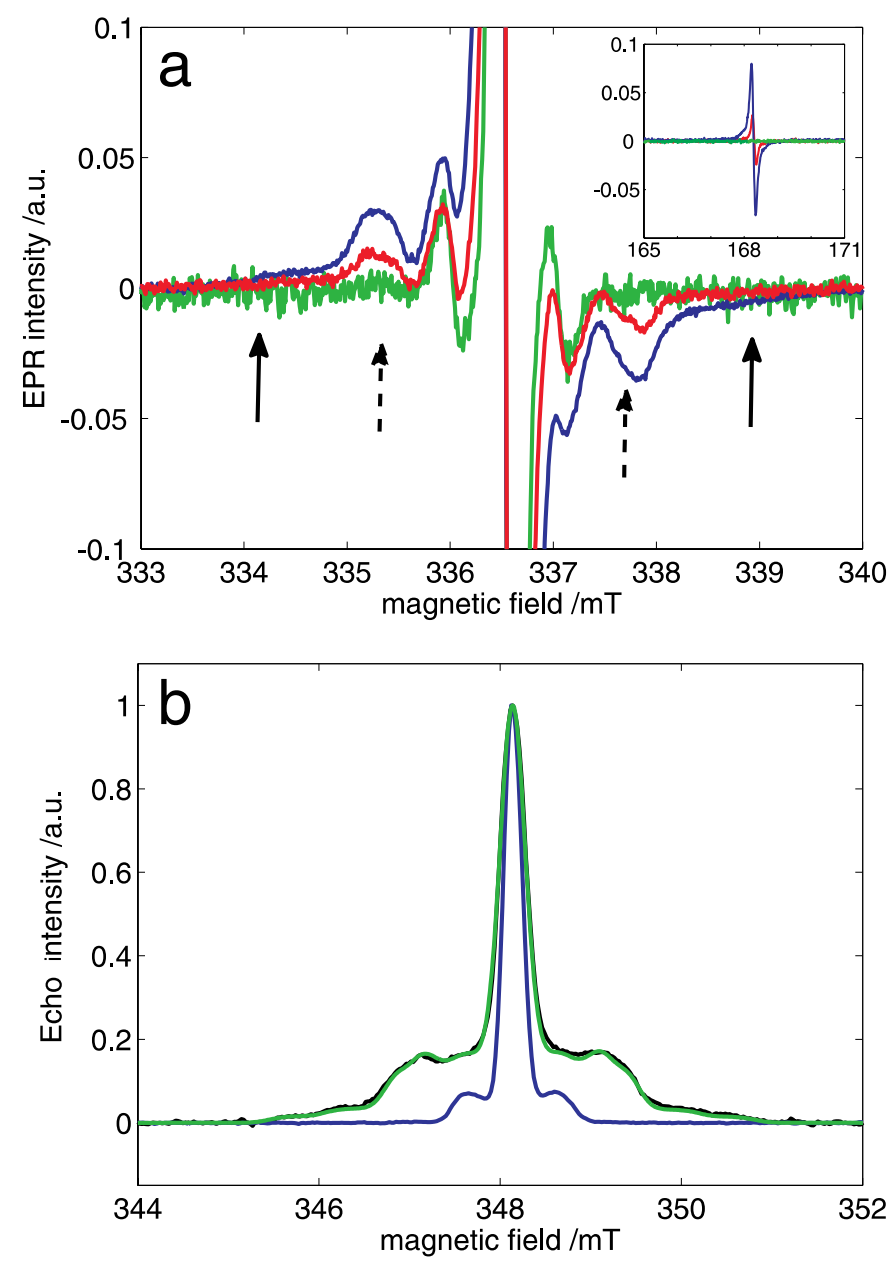

Figure 3. a. Expansion of the field region near $g=2.00$ of $X$-band $(9.437 \mathrm{GHz}) \mathrm{CW}$ EPR spectra measured $(T=20 \mathrm{~K}$, microwave power $250 \mathrm{nW}$ ) on $1.5 \mathrm{mM}$ (green), $15 \mathrm{mM}$ (red), and $30 \mathrm{mM}$ (blue) Finland radical solutions in glycerol:water (1:1) and $1 \mathrm{M}$ tretramethylammonium chloride. The arrows indicate the concentration-dependent features. The inset shows the half-field signal recorded at $2.8 \mathrm{~K}$ with a microwave power of $200 \mu \mathrm{W}$. b. ESE-detected EPR spectra of frozen $\mathrm{H}_{2} \mathrm{O} /$ glycerol solutions of $1.5 \mathrm{mM}$ (blue) and $30 \mathrm{mM}$ (black). A simulation matching the experimental $30 \mathrm{mM}$ spectra is shown in green. Expansions and simulation details are given in SI figure S4. Finland radical recorded at $9.766 \mathrm{GHz}$. The spectra were recorded at $7 \mathrm{~K}$. The spectra have been normalized to the central part of the signal and shifted to compensate for small differences in the microwave frequency in order to allow comparison of the side-band intensities. 


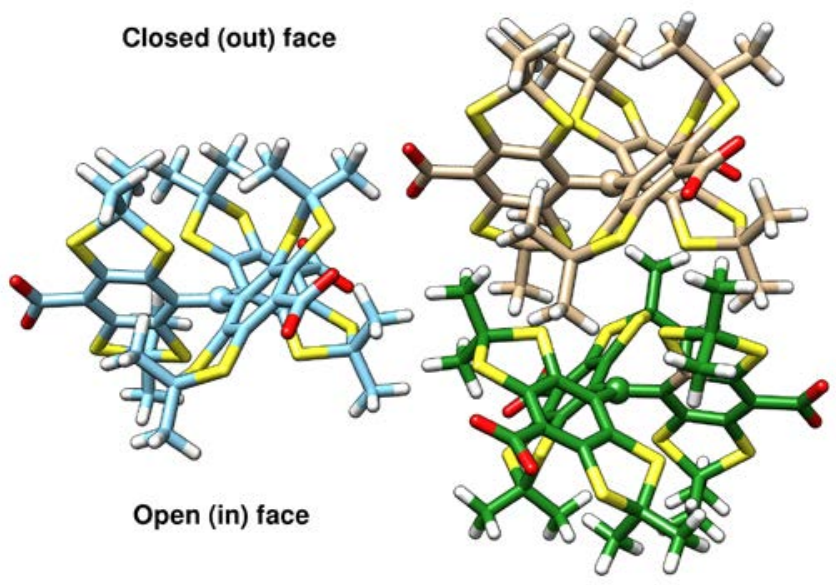

Figure 4. DFT minimum energy structure of the Finland radical (left), showing the non-equivalent faces, and of the PinMin Finland dimer (right). The central carbon of each radical is represented by a ball. Sodium counterions are omitted for the sake of clarity.

Extension of the dimer to form longer oligomers is possible by binding additional monomers with their "in" side facing the "out" side of an existing oligomer. On the contrary, oligomerization by interaction between preformed dimers seems improbable, since the interaction between the outer sides of the domes is rather weak. An additional monomer conformation in which both sides could participate in intermolecular contacts was found with $2 \mathrm{kcal} / \mathrm{mol}$ higher energy than the dome conformation and it could also contribute to the formation of larger Finland oligomers.

Time-dependent DFT calculations predict that the molar absorptivity of the dimer is about $15 \%$ lower than that expected from two non-interacting monomers but the position of the maxima is almost the same, in agreement with the observed concentration dependence of the absorptivity.

g-factors calculated with the B3LYP functional ${ }^{27}$ and the IGLO-II basis set ${ }^{28}$ for the BP/def2_SVP optimized geometries are 2.0032 for the monomer and 2.0034 for the PinMin dimer, in good agreement with the values obtained from the EPR spectra.

Calculations were not extended beyond the dimer. However, the formation of larger oligomers was experimentally observed in the gas phase and it agrees with the distribution of spin values inferred from magnetization studies described above.

Mass spectrometry experiments were performed under mild electrospray ionization (ESI) conditions in order to preserve non-covalent complexes. ${ }^{29}$ Finland radicals were dissolved in pure water and directly injected into the spectrometer.
Table 1. Calculated Finland dimers

\begin{tabular}{|c|c|c|c|}
\hline $\begin{array}{c}\text { Dimer }^{\mathrm{a}, \mathrm{b}} \\
\text { (Symmetry) }\end{array}$ & $\begin{array}{l}\text { Stabilization energy } \\
\left(\mathrm{kcal}_{\mathrm{mol}}{ }^{-1}\right)\end{array}$ & $\begin{array}{c}\text { Inter- } \\
\text { radical } \\
\text { distance } \\
(\AA)\end{array}$ & $\begin{array}{c}\text { Dimer } \\
\text { staggering }\end{array}$ \\
\hline PinMin $\left(\mathrm{S}_{6}\right)$ & 33.6 & 6.6 & 60 \\
\hline PinPin $\left(D_{3}\right)$ & 28.2 & 6.7 & 35 \\
\hline PoutMin $\left(\mathrm{C}_{3}\right)$ & 16.0 & 8.4 & 49 \\
\hline PoutMout $\left(\mathrm{S}_{6}\right)$ & 6.0 & 11.6 & 60 \\
\hline PoutPout $\left(\mathrm{D}_{3}\right)$ & 6.4 & 11.7 & 5 \\
\hline
\end{tabular}

Representative spectra are shown in Figure 5 and in the supplementary information (Figures S7-S14). Monomeric species appear as isotopic clusters with monoisotopic masses of 1066, and 1088, corresponding to the species $[\mathrm{FH}]^{+}$and $[\mathrm{FNa}]^{+}$ where $\mathrm{F}$ stands for the electrically neutral Finland trisodium salt. The positive charge is provided by an additional proton or sodium cation. A strong signal at $\mathrm{m} / \mathrm{z} 1077$ corresponds to a doubly charged Finland dimer $\left[\mathrm{F}_{2} \mathrm{NaH}\right]^{2+}$.

Ions with the same $\mathrm{m} / \mathrm{z}$ ratio but differing in total charge show different mobility in the gas phase. ${ }^{30}$ Figure $5 b$ shows a slice along the ion-mobility dimension of peaks with m/z 1060-1085 and Figure 5c shows enlarged views of the mass spectra for the different species. The separation between consecutive isotopic peaks clearly shows that the three ion-mobility peaks correspond to single charged monomer, double charged dimers and triple charged trimers, respectively.

Additional oligomer species were detected, although with lower intensity, at higher $\mathrm{m} / \mathrm{z}$ and with charges at least from one to five, according to $\mathrm{m} / \mathrm{z}$ separation of isotopic peaks. In particular, single charged species corresponding to dimers and trimers were observed at $2153\left[\mathrm{~F}_{2} \mathrm{Na}\right]^{+}$, and $3218\left[\mathrm{~F}_{3} \mathrm{Na}\right]^{+}$. A Finland tetramer with three positive charges, compatible with $\left[\mathrm{F}_{4} \mathrm{NaNaH}\right]^{3+}$, appears at $\mathrm{m} / \mathrm{z} 1435$. Groups of peaks separated by $22 / n \mathrm{Da}$, where $n$ is the charge of the cluster were observed indicating the formation of species with identical charge but arising from various combinations of sodium cations and protons. The intensity of peaks from Finland oligomers increases with the concentration of radical injected in the massspectrometer (SI Figure S8). 


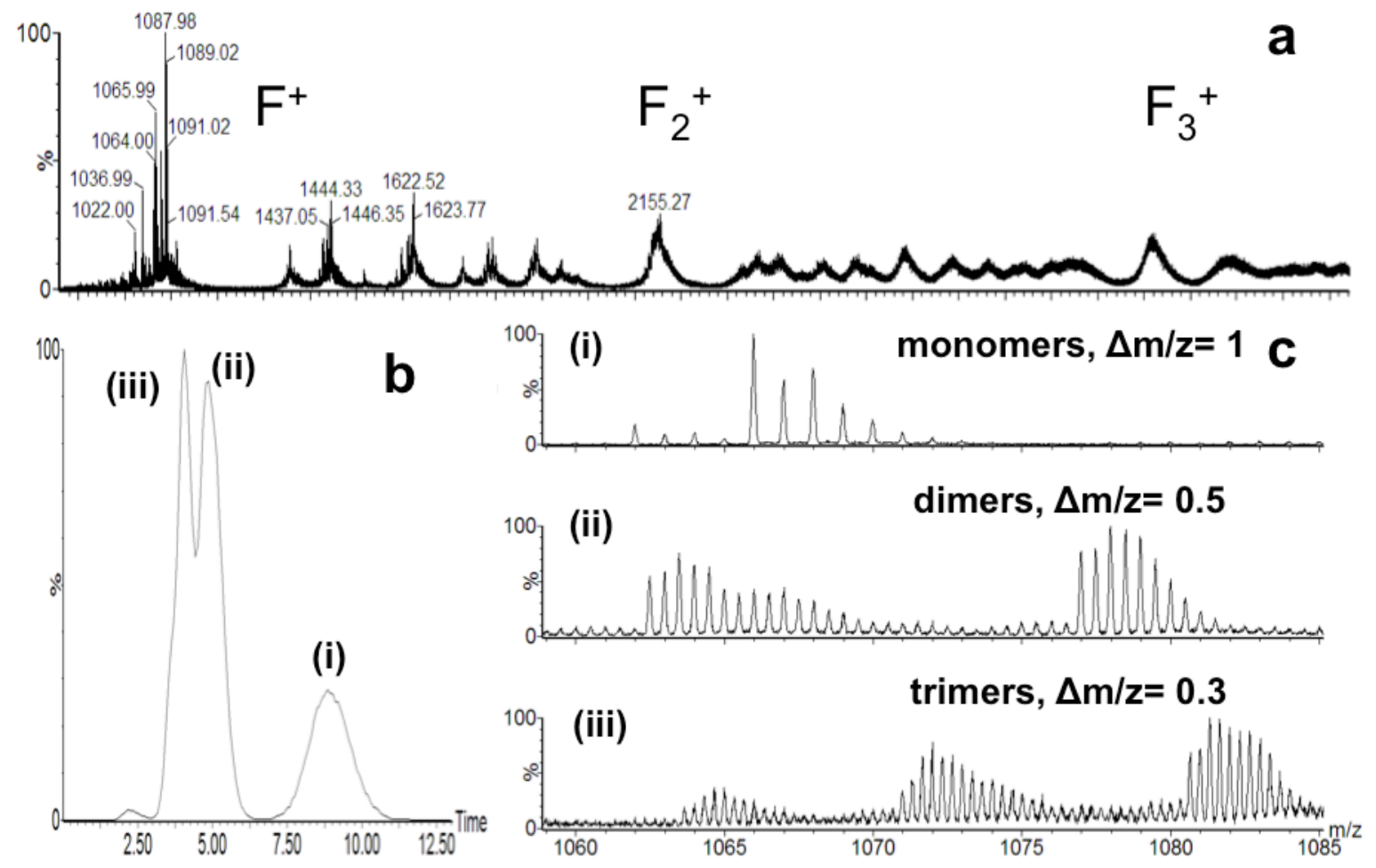

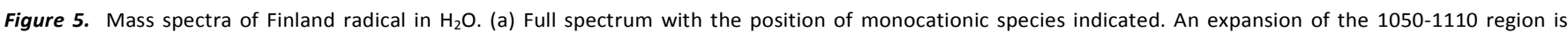

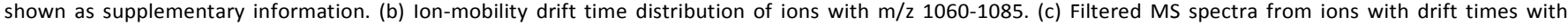

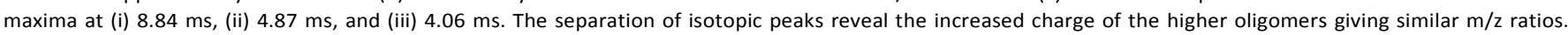

Nanometer-size supramolecular species were directly observed by cryo-EM of flash-frozen aqueous solutions of Finland radical. At pH 6.0 in $10 \mathrm{mM}$ 2-(N-morpholino) ethanesulfonic acid (MES) or $10 \mathrm{mM}$ citrate buffers spherical nanoparticles and fibres were clearly observed (Figure 6). Most spherical particles show uniform density, suggesting they are compact structures or hollow spheres with a small inner volume. A small number of larger spheres show low density in the centre suggesting the presence of an inner cavity. Two examples of nanospheres with low-density centres are shown in Figure 6. The size distribution of the compact spheres is approximately Gaussian. In MES buffer the same average diameter (10.2 \pm 0.2 $\mathrm{nm}$ ) was observed when the Finland concentration was increased from $15 \mathrm{mM}$ to $30 \mathrm{mM}$ but the number of particles observed was approximately doubled. In citrate buffer, the average diameter increases from $7.9 \pm 0.1$ to $9.0 \pm 0.1 \mathrm{~nm}$ when the Finland concentration increases from $15 \mathrm{mM}$ to $30 \mathrm{mM}$ but a similar number of particles were observed (SI figure S15).

Spherical objects of similar size were observed in samples dried at room temperature and negatively stained with uranyl acetate, confirming that nanoparticles are not an artifact caused by flash-freezing of the sample (Figure S16)

The buffer-dependency of the nanosphere dimensions suggests that buffer molecules may participate in the assembly process. This is in agreement with previous observations indicating that OX63 dimers could encapsulate tetraalkylammonium salts. ${ }^{12}$
We speculate that the protonated tertiary amine in MES may be similarly interacting with Finland nanospheres.

At pH 7.5 the predominant species observed by cryo-EM are fibres or sheets but no spherical particles were observed (SI figure S17). The $\mathrm{pH}$ dependency of the formation of nanospheres confirms that the carboxylate groups are participating in the self aseembly process, either stabilizing the nanospheres by hydrogen bonding, or by destabilizing the lateral packing by electrostatic repulsion at the higher $\mathrm{pH}$. However, axial packing is preserved at $\mathrm{pH} 7.5$

Nanostructure formation requires the three-dimensional selfassembly of large number of Finland radicals. While DFT calculations predict the formation of assemblies by packing along the C3 axis, the formation of spheres requires lateral packing. Finland molecules present a triangular array of carboxylate groups that could form hydrogen bonds with three additional Finland molecules. Lateral assembly would only be possible if at least some of the carboxylate groups are protonated, consistent with the fact that spherical nanoparticles are not observed at $\mathrm{pH}$ 7.5. Lateral packing of triangular elements is the basis of geodesic spheres. Thus we suggest that this is the mechanism behind the formation of spherical nanoparticles.

The nearly uniform density of the observed particles suggests that the laterally assembled units are not individual Finland molecules but axially packed (short) oligomers. Lateral packing of long oligomers could explain the formation of fibers. 
Water soluble TAM radicals represent a vesatile supramolecular scaffold that can generate a range of selfassembled stuctures formed by persistent organic radicals. In a previous example ${ }^{16}$, hydrogen-bond stabilized dimer capsules including tetramethyl ammonium were observed. In the present study, dimers and longer oligomers are formed but, in addition, nanospheres, fibers and sheets preserving their radical character are observed. The type of structures that are formed depends on the type of substituents decorating the trityl backbone, the $\mathrm{pH}$ and buffer used, and the possible encapsulation of guests.

The self-assembled nanostructures formed by Finland radical display a large number of paramagnetic centres in regular arrays. Coupling between radicals is observed by magnetic and EPR data. The EPR signature of the self associated species of OX63 and Finland are qualitatively similar but are characterized by different dipolar couplings and intensities of the half-field lines (SI Figures S18-S19), suggesting similar, but not identical local geometries.

\section{Conclusions}

Finland radical self-assembly in water represents the first example of a new class of paramagnetic metal-free supramolecular materials. Discrete, isotropic, high-spin particles offer obvious opportunities in spintronics, either as spin polarizers in electron transport nanodevices ${ }^{3}$ or as magnetic dopants of conducting carbon based materials. ${ }^{7}$ Higher dimensionality structures, such as long fibers and sheets made of regularly spaced persistent radicals might open new opportunities to build materials showing long-range magnetic order. ${ }^{1}$ The versatility of TAM self-assembled structures suggests that the properties of these systems can be tuned in a number of ways, including simple modifications in the substituents decorating the trityl scaffold, changing the experimental conditions, such as $\mathrm{pH}$, or by explointing the hostguest properties, which could lead to the design of sensors.

The versatilty of TAM self-assembly may complicate the design as multiple supramolecular forms may be formed simultaneously. We are currently exploring conditions to either produce or isolate a single class of nanoparticles and
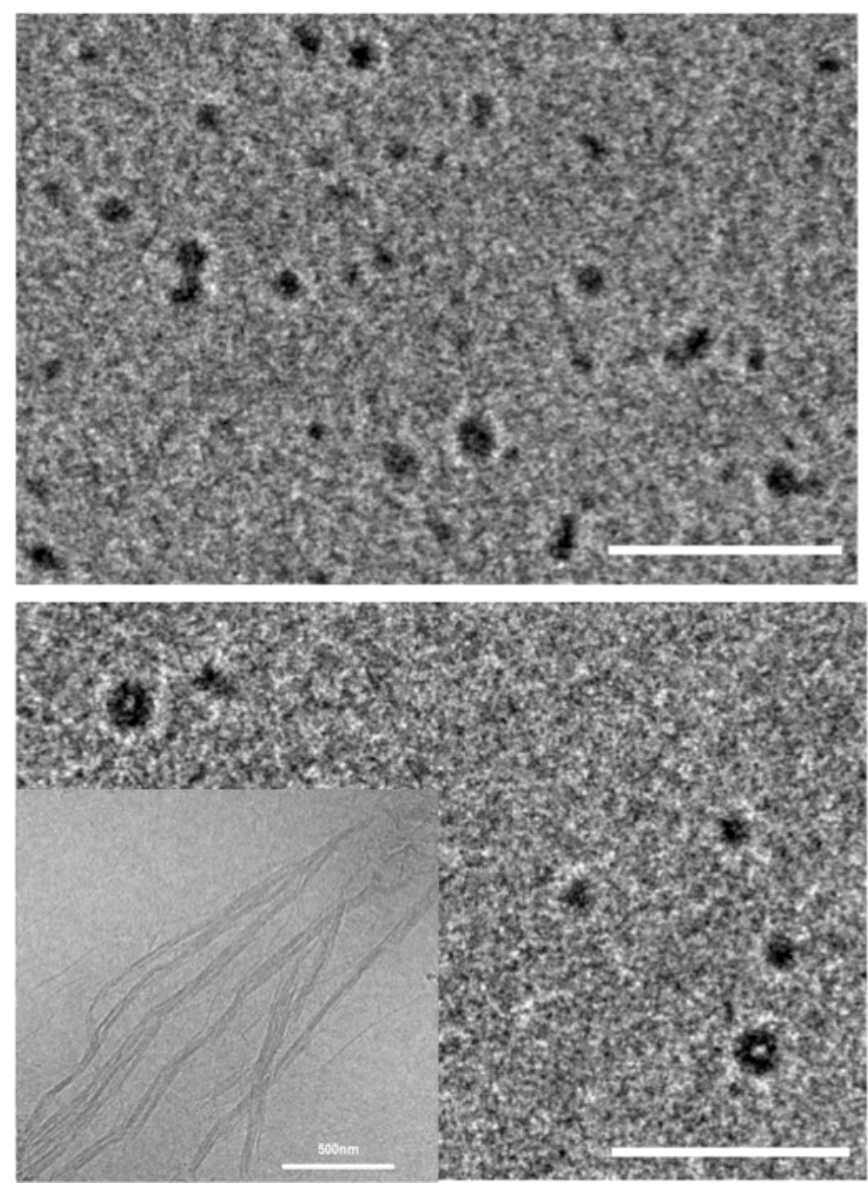

Figure 6. Cryo-Electron Microscopy images of Finland solutions. Top) $30 \mathrm{mM}$ Finland in $10 \mathrm{mM}$ citrate buffer, $\mathrm{pH}$ 6.0. Bottom) $30 \mathrm{mM}$ Finland in $10 \mathrm{mM}$ MES buffer, pH 6.0. Inset). Finland filaments in $10 \mathrm{mM}$ MES. Scale bars are $100 \mathrm{~nm}$ in the main panels and $500 \mathrm{~nm}$ in the inset.

investigating conditions enhancing the coupling between radicals within the nanoparticles in the quest for new organic high-spin nanospheres based on stable trityl radicals.

\section{Experimental}

Finland radical was from Oxford Instruments. UV spectra were measured in a JASCO V-650 instrument at $298 \mathrm{~K}$ and $\mathrm{pH} 7.0$ in carefully degassed $10 \mathrm{mM}$ phosphate buffer using cells with light paths from $1 \mathrm{~cm}$ to $0.01 \mathrm{~cm}$ Measurements were carried out in triplicate.

Magnetic measurements were performed with a commercial SQUID magnetometer (MPMS_XL from Quantum Design), which is equipped with high sensitivity and continuous cooling options.

X-band continuous-wave (CW) EPR spectra were recorded on a Bruker ESP300E spectrometer equipped with a liquid helium cryostat (Oxford Inc.), allowing operation from room temperature down to $2.5 \mathrm{~K}$. A modulation frequency of 100 
$\mathrm{kHz}$ was used. Details about temperature, microwave frequency and power are given in the figure caption. The samples were evacuated to 1 mbar to remove excess of $\mathrm{O}_{2}$. Microwave power was selected to avoid saturation effects as determined by the linear dependency of the intensity with the square root of the incident power.

Pulsed EPR experiments were performed on a Bruker ElexSys E580 spectrometer (mw frequency of $9.76 \mathrm{GHz}$ ) equipped with a liquid-helium cryostat (Oxford Inc.).

ESE (electron spin echo)-detected EPR experiments were performed using the $\pi / 2-\tau-\pi$ - $\tau$-echo microwave pulse sequence, with pulse lengths $t_{\pi / 2}=200 \mathrm{~ns}, t_{\pi}=400 \mathrm{~ns}$ and inter-pulse distance $\tau=1 \mu \mathrm{s}$.

PEANUT (phase-inverted echo-amplitude detected nutation) experiments ${ }^{21}$ were performed using the $\pi / 2-\tau$-(HTA $)_{\mathrm{x}}(\mathrm{HTA})_{-\mathrm{x}^{-}}$ $\tau$-echo. The length of the first high turning angle (HTA) pulse with phase 0 had length $t$, while the second HTA pulse had a length $T$ - $t$ with $T$ set to 2060 ns and $t$ varied in steps of 4ns. The time axis was doubled in order to obtain the PEANUT peaks at the nutation frequency instead of double this frequency. The pulse lengths $t_{\pi / 2}=16 \mathrm{~ns}$ and $\tau=200 \mathrm{~ns}$. The mw field strength $v_{1}$ was taken to be $3.5 \mathrm{MHz}$. The time traces were baselinecorrected with a third-order polynomial, apodized with a symmetrized Hanning window such as to emphasize only the central part of the time trace and zero-filled before Fourier transformation.

Ion mobility mass spectrometry experiments were performed on a Synapt G1 HDMS instrument (Waters, Manchester, UK) vacuum-modified for optimal transmission of non-covalent ions. Ions were produced by nanoESI with a Triversa Nanomate system (Advion BioSciences, Ithaca, NY, USA).

Density Functional Theory (DFT) calculations were performed with the Orca 3.0.0 package ${ }^{22}$ The gradient-corrected BeckePerdew functional ${ }^{23}$ (BP86) was chosen together with the Ahlrichs polarized split-valence basis set (Def2-SVP in Orca notation), ${ }^{24}$ and the resolution of identity approximation. A recent version of the empirical dispersion correction by Grimme was included, ${ }^{31}$ and the basis set superposition error was minimized by using the geometrical counterpisie correction. ${ }^{32}$ The conductor like screening model developed by $\mathrm{Klamt}^{25}$ (COSMO) was used to introduce solvent effects, with the values 53 for the permitivity and 1.4 for the refraction index. These are approximate values for a 50\% glycol-water mixture at low temperature. Graphics were prepared using Chimera. $^{33}$

Cryo-EM images were recorded on a Tecnai G2 F20 (FEI) 200 $\mathrm{kV}$ FEG TEM cryomicroscope with Eagle 4kx4k CCD and tomograph. The $5 \mu \mathrm{l}$ samples were prepared in a Holey coal grid with ultraviolet light pretreatment using cryofixation by Vitrobot Mark III (FEI). Samples of 15mM and 30mM Finland concentrations were prepared in 10mM buffer (MES or citrate), degassed and filtered under vacuum with a $0.2 \mu \mathrm{m}$ pore filter. Samples were stored in liquid nitrogen before they were observed at electronic microscope ${ }^{34}$.

\section{Acknowledgements}

We thank Dr. Carmen Lopez-Iglesias for her help with cryoelectron microscopy. We acknowledge the use of the facilities from the NMR ICTS and the "Centres Científics i Tecnològics" of the University of Barcelona and from "Servicio General de Apoyo a la Investigación-SAI” of the Universidad de Zaragoza, and the NMR ICTS. This work was supported in part by funds from the Spanish government (BIO2013-45793-R, MAT201238318-C03-01, and Juan de la Cierva), Cataluña (2009SGR1352 and Beatriu de Pinós) and Aragón (E98-MOLCHIP) governments, The European Comission (BioNMR contract 261863), and the COST action (TD1103).

\section{Notes and references}

${ }^{a}$ Biomolecular NMR laboratory. Department of Organic Chemistry. University of Barcelona . Cluster Building. Barcelona Science Park. Baldiri Reixac, 10-12 08028 Barcelona. Spain.

${ }^{b}$ Department of Physical Chemistry. University of Barcelona. Martí i Franquès, 1-11 08028-Barcelona. Spain.

${ }^{c}$ Institute of Theoretical and Computational Chemistry. University of Barcelona (IQTCUB).

${ }^{d}$ Mass Spectrometry Core Facility. Institute for Research in Biomedicine (IRB Barcelona). Baldiri Reixac, 10-12 08028 Barcelona. Spain.

e Instituto de Ciencia de Materiales de Aragón, CSIC-Universidad de Zaragoza, Pedro Cerbuna 12, 50009 Zaragoza, Spain

${ }^{f}$ Department of Physics. University of Antwerp. Universiteitsplein 1 B2610 Wilrijk. Belgium.

Electronic Supplementary Information (ESI) available: Detailed experimental information and computational methods. Representative UV, EPR, and MS spectra. Dimer structures listed in table 1.

1 A. Rajca, J. Wongsriratanakul, S. Rajca, Science, 2001, 294, 1503-1505; I. Ratera, J. Veciana, Chem. Soc. Rev. 2012, 41, 303-349.

2 G. M. Clore, J. Iwahara, Chem. Rev. 2009, 109, 4108-4139.

3 B. O. Jahn, H. Ottosson, M. Galperin, J. Fransson, ACS Nano 2013, 7, 1064-1071; R. Frisenda, R. Gaudenzi, C. Franco, M. MasTorrent, C. Rovira , J. Veciana, I. Alcon, S. T. Bromley, E. Burzurí, and H. S. J. van der Zant, Nano Lett. 2015, 15, 3109-3114;

4 A. Rajca, A. Olankitwanit, Y. Wang, P. J. Boratyński, M. Pink, and S. Rajca, J. Am. Chem. Soc., 2013, 135, 18205-18215.

5 M.Mannini, L. Sorace, L- Gorini, F. M. Piras, A. Caneschi, A. Magnani, S. Menicchetti, D. Gatteschi. Langmuir, 2007, 23, 23892397.

6 O. Shekhah, N. Roques, V. Mugnaini, C. Munuera, C. Ocal, J. Veciana, C. Wöll, Langmuir, 2008, 24, 6640-6648.

7. J. Choi, H. Lee, K. Kim, B. Kim, S. Kim Phys. J. Phys. Chem. Lett. 2009, 1, 505-5098; S-A. Savu, I. Biswas, L. Sorace, M. Mannini, D. Rovai, A. Caneschi, T. Chassé, M.B. Casu Chemistry-A European Journal 2013, 19, 3445-3450; J. Hong, E. Bekyarova, P. Liang, W.A. deHeer, R.C. Haddon, S. Khrizroev Scientific Reports, 2012, 624.

8 Y. Ying, R.K. Saini, F. Liang, A. K. Sadana, W.E. Billups. Org. Lett. 2003, 5, 1471-1473. 
9 L. Adriaenssens and P. Ballester, Chem. Soc. Rev., 2013, 42, 32613277; M. M. Conn and J. Rebek, Chem. Rev., 1997, 97, 1647-1668; M. D. Pluth and K. N. Raymond, Chem. Soc. Rev., 2007, 36, 161171.

10 J. Sedó, N. Ventosa, M. A. Molins, M. Pons, C. Rovira, J. Veciana, J. Org. Chem., 2001, 66, 1579-1589.

11 M.C. Krishna, S. English, K. Yamada, J. Yoo, R. Murugesan, N. Devasahayam, J.A. Cook, K. Golman, J.H. Ardenkjaer-Larsen, S. Subramanian, J.B. Mitchell, Proc. Natl. Acad. Sci. USA 2002, 99 2216-2221.

12 S. Subramanian and M. C. Krishna, Biol. Magn. Reson. 2005, 23 (Part A), 321-382; C. Rizzi, A. Samouilov, V. K. Kutala, et al., Free Radicals Biol. Med. 2003, 35, 1608-1618.

13 R. G. Griffin and T. F. Prisner, Phys. Chem. Chem. Phys., 2010, 12, 5737 and following articles in a DNP themed issue.

14 J. H. Ardenkjær-Larsen, B. Fridlund, A. Gram, G. Hansson, L. Hansson, M. H. Lerche, R. Servin, M. Thaning and K. Golman, Proc. Natl. Acad. Sci. U. S. A. 2003, 100, 10158-10163.

15 S. J. Nelson, J. Kurhanewicz et al. Sci. Transl. Med. 2013, 5, 198 ra108.

16 I. Marin-Montesinos, J. C. Paniagua, M. Vilaseca, A. Urtizberea, F. Luis, M. Feliz, F. Lin, S. Van Doorslaer and M. Pons, Phys. Chem. Chem. Phys. 2015, 17, 5785-5794.

17 C.R. Cantor, P.R. Schimmel, Biophysical Chemistry (II). Techniques for the study of biological structure and function. W.H. Freeman and co. San Francisco 1980 pp.349-408.

18 F. Luis, J.M. Torres, L.M. García, J. Bartolomé, J. Stankiewicz, F. Petroff, F. Fettar, J.L. Maurice, A. Vaurès, Phys. Rev B 2002, 65, 094409 (1-10).

19 M. K. Bowman, C. Mailer, and H. J Halpern, J. Magn. Reson. 2005, 172, 254-267; R. Owenius, G. R. Eaton, and S. S. Eaton, J. Magn. Reson. 2005, 172, 168-175.

20 S. N. Trukhan, V.F. Yudanov, V.M. Tormyshev, O.Yu. Rogozhnikova, D.V. Trukhin, M.K Bowman, M.D. Krzyaniak, H. Chen, O.N. Martyanov, J. Magn. Reson. 2013, 233, 29-35.

21 S. Stoll, G. Jeschke, M. Willer, A. Schweiger, J. Magn. Reson. 130, 86-96 (1998)

22 F. Neese, Wiley Interdiscip. Rev.: Comput. Mol. Sci. 2012, 2, 73-78.

23 A. D. Becke, Phys. Rev. A 1988, 38, 3098-3100; P86; J. P. Perdew, Phys. Rev. B 1986, 33, 8822-8824.

24 A. Schaefer, H. Horn, and R. Ahlrichs, J. Chem. Phys. 1992, 97, 2571-2577.

25 S. Sinnecker, A. Rajendran, A. Klamt, M.Diedenhofen, and F. Neese, J. Phys. Chem. A 2006, 110, 2235-2245.

26 S. Wojyulewski and S. Grabowski, Chem.Phys.Lett, 2003,378,388.

27 A. D. Becke, J. Chem. Phys. 1993, 98, 5648-5652; C. Lee, W. Yang, and R. G. Parr, Phys. Rev. B 1988 37, 785-789; B. Miehlich, A. Savin, H. Stoll, and H. Preuss, Chem. Phys. Lett. 1989, 157, 200-206.

28 W. Kutzelnigg, U. Fleischer and M. Schindler The IGLO-Method: Ab Initio Calculation and Interpretation of NMR Chemical Shifts and Magnetic Susceptibilities, Springer-Verlag, 1990, vol. 23.

29 Z. Qi, T.Heinrich, S. Moorthy, and C.A. Schalley, Chem. Soc. Rev. 2015, 44, 515-531.

30 D. E. Clemmer and M. F. J. Jarrold, Mass Spectrometry 1997, 32, 577.
31 S. Grimme, J. Antony, S. Ehrlich and H. Krieg, J. Chem. Phys. 2010, 132, 154104-154122; S. Grimme, S. Ehrlich and L. Goerigk, J. Comput. Chem, 2011, 32, 1456-1465.

32 H. Kruse, S. Grimme, J. Chem. Phys. 2012, 136, 154101-154116.

33 E.F. Pettersen, T.D. Goddard, C.C. Huang, G.S. Couch, D.M. Greenblatt, E.C Meng, T.E. Ferrin. J Comput Chem. 2004, 25, 16051612.

34 W. F. Tivol, A. Briegel and G. J. Jensen, Microsc. Microanal., 2008,14, 375-379. 